\title{
Article
}

\section{Optimal Saving by Expected Utility Operators}

\author{
Irina Georgescu ${ }^{1, * \mathbb{C}}$ and Jani Kinnunen ${ }^{2}$ \\ 1 Academy of Economic Studies, Department of Economic Cybernetics, Piaţa Romana No 6 R 70167, Oficiul \\ Postal 22, 010552 Bucharest, Romania \\ 2 Department of Information Systems, Åbo Akademi University, Tuomiokirkkotori 3, 20500 Turku, Finland; \\ jani.kinnunen@abo.fi \\ * Correspondence: irina.georgescu@csie.ase.ro; Tel.: +0040-21-7456-082
}

Received: 5 December 2019; Accepted: 28 January 2020; Published: 9 February 2020

\begin{abstract}
This paper studies an optimal saving model in which risk is represented by a fuzzy number and the total utility function of the model is defined by an expected utility operator. This model generalizes some existing possibilistic saving models and from them, by a particularization, one can obtain new saving models. In the paper, sufficient conditions are set for the presence of potential risk to increase optimal saving levels and an approximation formula for optimal saving is demonstrated. Particular models for a few concrete expected utility operators are analyzed for triangular fuzzy numbers and CRRA-utility functions.
\end{abstract}

Keywords: expected utility operator; possibilistic saving models; precautionary saving

\section{Introduction}

The effect of different types of risk on investment decisions is an intensely studied topic in economic and financial literature. To cope with a possible loss, the investor can transfer some of his current wealth to the future. In the case of saving models, the study of the influence of risk on a consumer's decisions to transfer more or less of his current wealth to the future began with the works of Leland [1], Sandmo [2] and Drèze and Modigliani [3]. They investigated the way the presence of a labor-income risk influences the optimal saving level. Approximately at the same time, Sandmo [2] and Rothschild and Stiglitz [4] researched the changes produced by an interest-rate risk as a measure of the optimal saving variation when passing from a certain saving model to a saving model with risk. If the precautionary saving is positive then the risk presence makes the optimal saving level rise (in other terms, risk generates extra saving). According to [1-3], the labor-income risk generates extra saving if and only if the third derivative is equivalent with "consumer prudence", expressed by the prudence index associated with the utility function. A theorem of Rothschild and Stiglitz [4] shows that the presence of an interest-rate risk generates extra saving if and only if the relative prudence index [5] of the utility function is higher that 2. Later on, the theory of the saving model was developed by Eeckhoudt and Schlesinger [6], Li [7], Baiardi et al. [8], etc. An intuitive exposure of the saving model theory can be found in Section 6 of the monograph [9] and the state of art dedicated to the last results on this topic is contained in the recent paper of Baiardi et al. [10].

The saving models from all above mentioned papers are built in the context of von Neumann-Morgenstern Eu- theory, risk being modeled by random variables. One can meet situations of uncertainty for which the probabilistic risk is not appropriate (for example, in the absence of an insufficiently large dataset). One can refer then to Zadeh's possibility theory [11], therefore uncertainty is modeled by possibilistic distributions (in particular, by fuzzy numbers) (see [12-14]).

Fuzzy numbers are the most important class of possibilistic distributions. They have algebraic properties close to those of real numbers, which make the risk representation by fuzzy numbers lead to effective models [12-18]. 
Papers [19,20] propose some possibilistic optimal saving models, in which different types of risk are represented by a fuzzy number. The construction of this model is based on a notion of "possibilistic expected utility" (defined in [12], Definition 4.2.7 or [21]) and the possibilistic indicators associated with a fuzzy number $[12,13,15]$.

On the other hand, there exist several possibilistic expected utilities (by [12], Section 4.2). The expected utility operators introduced in [12] allow to define a general notion of possibilistic expected utility which comprises the already existing ones. All the expected utility operators provide the same notion of possibilistic expected value, but distinct possibilistic variances.

This paper studies a saving model in the possibilistic context defined by an expected utility operator. The model in the paper extends the one from [20] and, by particularization, other concrete models of optimal saving can be obtained.

A saving model can be built in the context of EU theory associated with each expected utility operator, but the analysis of solutions of the optimization problem associated with the model requires the use of $D$-operators introduced in [19].

We present shortly the structure of the paper. Section 2 presents briefly the two notions of possibilistic expected utility from [21,22] as well as corresponding possibilistic indicators. In Section 3 there are recalled the expected utility operators and the $D$-operators from $[19,21,22]$. Section 4 contains the construction of the saving model associated with an expected utility operator: The basic entities, the definition of total utility function, the maximization problem and the first order condition associated with it. In Section 5 there is proved an approximation calculation formula of the optimal saving level. An order relation is introduced on the set of expected utility operators and the behaviour of optimal saving towards this order is studied. In Section 6 the notion of T-precautionary saving is defined, indicator which expresses the variation of optimal saving determined by the presence of a possibilistic risk represented by a fuzzy number. Among the results of the section, we mention a sufficient condition such that the presence of possibilistic risk should lead to an increase in optimal saving. Section 7 presents particular saving models for triangular fuzzy numbers and for $C R R A$-utility functions. The paper ends with a section of concluding remarks.

\section{Two Possibilistic Expected Utilities}

The classical von Neumann-Morgenstern (= EU theory) is the framework in which the topics of probabilistic risk have been developed. The notion of expected utility lies in the center of this EU theory: If $X$ is a random variable and $u: \mathbf{R} \rightarrow \mathbf{R}$ is a continuous utility function then the mean value $M(u(X))$ of the random variable $u(X)$ is the expected utility associated with $X$ and $u$.

To define a possibilistic expected utility we need three entities:

- A weighting function $f:[0,1] \rightarrow \mathbf{R}(f$ is a non-negative and increasing function that satisfies $\left.\int_{0}^{1} f(\gamma) d \gamma=1\right)$

- $\quad$ a fuzzy number $A$ with the level sets $[A]^{\gamma}=\left[a_{1}(\gamma), a_{2}(\gamma)\right], \gamma \in[0,1]$;

- $\quad$ a utility function $u: \mathbf{R} \rightarrow \mathbf{R}$ of class $C^{2}$.

In monograph [12] there are defined two notions of possibilistic expected utilities:

$$
\begin{gathered}
E_{1}(f, u(A))=\frac{1}{2} \int_{0}^{1}\left[u\left(a_{1}(\gamma)\right)+u\left(a_{2}(\gamma)\right)\right] f(\gamma) d \gamma \\
E_{2}(f, u(A))=\int_{0}^{1}\left[\frac{1}{a_{2}(\gamma)-a_{1}(\gamma)} \int_{a_{1}(\gamma)}^{a_{2}(\gamma)} u(x) d x\right] f(\gamma) d \gamma
\end{gathered}
$$

Particularizing $u(x)$ in Formulas (1) and (2) we find some indicators associated with the fuzzy number $A$. 
For $u=1_{\mathbf{R}}$ (the identity function of $\mathbf{R}$ ), the two possibilistic expected utilities define the same notion of possibilistic expected value $[13,15]$ :

$$
E_{f}(A)=E_{1}\left(f, 1_{\mathbf{R}}(A)\right)=E_{2}\left(f, 1_{\mathbf{R}}(A)\right)=\frac{1}{2} \int_{a_{1}(\gamma)}^{a_{2}(\gamma)}\left[a_{1}(\gamma)+a_{2}(\gamma)\right] f(\gamma) d \gamma
$$

Setting $u(x)=\left(x-E_{f}(A)\right)^{2}$ in Formulas (1) and (2) one obtains two distinct notions of possibilistic variance $[12,13,15]$ :

$$
\begin{gathered}
\operatorname{Var}_{1}(f, A)=\frac{1}{2} \int_{0}^{1}\left[\left(u\left(a_{1}(\gamma)\right)-E_{f}(A)\right)^{2}+\left(u\left(a_{2}(\gamma)\right)-E_{f}(A)\right)^{2}\right] f(\gamma) d \gamma \\
\operatorname{Var}_{2}(f, A)=\int_{0}^{1}\left[\frac{1}{a_{2}(\gamma)-a_{1}(\gamma)} \int_{a_{1}(\gamma)}^{a_{2}(\gamma)}\left(x-E_{f}(A)\right)^{2} d x\right] f(\gamma) d \gamma
\end{gathered}
$$

\section{Expected Utility Operators and D-Operators}

The two possibilistic utilities $E_{1}(f, u(A))$ and $E_{2}(f, u(A))$ introduced in the previous section define two different possibilistic EU theories.

Inside each EU theory defined by $E_{1}(f, u(A))$ and $E_{2}(f, u(A))$ can be developed different topics of risk theory. The proposed notions, the risk indicators and the obtained results corresponding to the two approaches will be in general different. As examples, we will mention the two risk aversion theories corresponding to $E_{1}(f, u(A))$ and $E_{2}(f, u(A))$ (see [12], Sections 4.3 and 4.4). Analogously, we will obtain two different treatments of optimal saving.

The expected utility operators, introduced in [21] offer a natural framework to develop a general possibilistic Eu- theory, which should include the two mentioned above. Thus in this framework, it could be elaborated in Chapter 5 of [12] a possibilistic risk aversion associated with each expected utility operator. In the same stream, in this paper, we will build a possibilistic optimal saving model for each expected utility operator.

In this section we will recall from $[12,21,22]$ the definition of expected utility operators, some of their properties and some examples.

Let $\mathcal{F}$ be the set of fuzzy numbers and $\mathcal{C}(\mathbf{R})$ the set of continuous functions from $\mathbf{R}$ to $\mathbf{R}$. Let $\mathcal{U}$ be a subset of $\mathcal{C}(\mathbf{R})$ satisfying the following properties:

$\left(U_{1}\right) \mathcal{U}$ contains the constant functions and first and second degree polynomial functions;

$\left(U_{2}\right)$ If $a, b \in \mathbf{R}$ and $g, h \in \mathcal{U}$ then $a g+b h \in \mathcal{U}$.

For any $a \in \mathbf{R}$, we denote by $\bar{a}: \mathbf{R} \rightarrow \mathbf{R}$ the function $\bar{a}(x)=a$, for any $x \in \mathbf{R}$.

We fix a family $\mathcal{U}$ with the properties $\left(U_{1}\right),\left(U_{2}\right)$ and a weighting function $f: \mathbf{R} \rightarrow \mathbf{R}$.

Definition 1 ([21]). An ( $f$-weighted) expected utility operator is a function $T: \mathcal{F} \times \mathcal{U} \rightarrow \mathbf{R}$ such that for any $a, b \in \mathbf{R}, g, h \in \mathcal{U}$ and $A \in \mathcal{F}$ the following conditions are fulfilled:

$$
\begin{aligned}
& \text { (a) } T\left(A, 1_{\mathbf{R}}\right)=E_{f}(A) ; \\
& \text { (b) } T(A, \bar{a})=a ; \\
& \text { (c) } T(A, a g+b h)=a T(A, g)+b T(A, h) ; \\
& \text { (d) } g \leq h \text { implies } T(A, g) \leq T(A, h) .
\end{aligned}
$$

Several times in the paper we will write $T(A, g(x))$ instead of $T(A, g)$.

The $T$-variance $\operatorname{Var}_{T}(A)$ associated with an expected utility operator $T$ is defined by $\operatorname{Var}_{T}(A)=$ $T\left(A,\left(x-E_{f}(A)\right)^{2}\right)$.

Example 1. Let $T_{1}: \mathcal{F} \times \mathcal{C}(\mathbf{R}) \rightarrow \mathbf{R}$ defined by

$$
T_{1}(A, g)=\frac{1}{2} \int_{0}^{1}\left[g\left(a_{1}(\gamma)\right)+g\left(a_{2}(\gamma)\right)\right] f(\gamma) d \gamma=E_{1}(f, g(A))
$$


for any $g \in \mathcal{C}(\mathbf{R})$ and for any fuzzy number $A$ whose level sets are $[A]^{\gamma}=\left[a_{1}(\gamma), a_{2}(\gamma)\right], \gamma \in[0,1]$.

Example 2. Let $T_{2}: \mathcal{F} \times \mathcal{C}(\mathbf{R}) \rightarrow \mathbf{R}$ defined by

$$
T_{2}(A, g)=\int_{0}^{1}\left[\frac{1}{a_{2}(\gamma)-a_{1}(\gamma)} \int_{a_{1}(\gamma)}^{a_{2}(\gamma)} g(x) d x\right] f(\gamma) d \gamma=E_{2}(f, g(A))
$$

for any $g \in \mathcal{C}(\mathbf{R})$ and for any fuzzy number $A$ whose level sets are $[A]^{\gamma}=\left[a_{1}(\gamma), a_{2}(\gamma)\right], \gamma \in[0,1]$.

If $T$ is an expected utility operator then $T(A, u)$ is called generalized possibilistic expected utility of the fuzzy number $A$ w.r.t. the weighting function $f$ and the utility function $u$. By Examples 1 and 2, the possibilistic expected utilities $E_{1}(f, g(A))$ and $E_{2}(f, g(A))$ correspond to the expected utility operators $T_{1}$ and $T_{2}$.

Proposition 1 ([21]). Let $T$ be an expected utility operator. For any fuzzy number $A$ and for any $u \in \mathcal{U}$, the following approximation formula holds:

$$
T(A, u) \approx u\left(E_{f}(A)\right)+\frac{u^{\prime \prime}\left(E_{f}(A)\right)}{2} \operatorname{Var}_{T}(A)
$$

The $D$-expected utility operators are a class of expected utility operators. They have been introduced in [22] to obtain approximate solutions of a portfolio choice problem formulated in EU theory associated with a $D$-operator.

In the rest part of this section, we will recall from [22] the definition of the $D$-operators and a few of their properties.

Let $g(x, \lambda): \mathbf{R}^{2} \rightarrow \mathbf{R}$ be a real function in the arguments $x$ and $\lambda$. With respect to such a function $g(x, \lambda)$ we consider the following properties:

(i) $\quad g(x, \lambda)$ is continuous with respect to $x$ and partially derivable with respect to parameter $\lambda$;

(ii) For any parameter $\lambda \in \mathbf{R}$, the function $\frac{\partial g(., \lambda)}{\partial \lambda}: \mathbf{R} \rightarrow \mathbf{R}$ is continuous.

Definition 2 ([22]). An expected utility operator $T: \mathcal{F} \times \mathcal{C}(\mathbf{R}) \rightarrow \mathbf{R}$ is derivable with respect to parameter $\lambda$ (shortly, $T$ is a D-operator) if for any fuzzy number $A$ and for any function $g(x, \lambda)$ with the properties (i) and (ii), the following axioms are verified:

$\left(D_{1}\right)$ The function $\lambda \longmapsto T(A, g(., \lambda))$ is derivable (with respect to $\lambda$ ).

$\left(D_{2}\right) T\left(A, \frac{\partial g(., \lambda)}{\partial \lambda}\right)=\frac{d}{d \lambda} T(A, g(., \lambda))$.

Proposition 2 ([22]). The expected utility operators from Examples 1 and 2 are D-operators.

Proposition 3 ([21]). If $T$ is an expected utility operator and $g: \mathbf{R} \rightarrow \mathbf{R}$ is a convex function then $g\left(E_{f}(A)\right) \leq$ $T(A, g)$.

\section{The T-Model of Optimal Saving}

In this section we will define a model of optimal saving inside EU theory associated with an expected utility operator. Our construction has as a starting point the probabilistic model of precautionary saving from [9] (p. 95) and the possibilistic model of optimal saving from [20].

To fix ideas, we will briefly present the probabilistic model from Section 6.2 of [9]. This is a two-period consumption model, defined by the following entities:

- two utility functions $u(y)$ and $v(y): u(y)$ is the consumer's utility function in the first period 0 , and $v(y)$ is the consumer's utility function in the second period 1 ;

- $\quad$ in period 0 there is a sure income $y_{0}$, and in period 1 there is an uncertain income described by a random variable $\tilde{y}$; 
- $\quad s$ is the level of saving for period 0 ; to face the risk in period 1, the consumer transfers to this period a part $s$ of $y_{0}$;

- $\quad r$ is the interest rate for saving.

The utility functions $u$ and $v$ are of class $C^{2}$ and fulfill the conditions $u^{\prime}>0, v^{\prime}>0, u^{\prime \prime}<0$, $v^{\prime \prime}<0$.

The consumer wishes to choose that value $s$ maximizing the following total utility function:

$$
U(s)=u\left(y_{0}-s\right)+M(v((1+r) s+\tilde{y})
$$

By Section 6.2 of [9], $U$ is a strictly concave function.

We will consider the optimization problem $\max _{s} U(s)=U\left(s^{\circ}\right)$, in which $s^{\circ}$ is the optimal saving of this probabilistic model. The first-order condition associated with the optimization problem is:

$$
-u^{\prime}\left(y_{0}-s\right)+(1+r) M\left(v^{\prime}((1+r) s+\tilde{y})\right)=0
$$

As seen from (6), the total utility $U(s)$ has two components:

(a) the utility $u\left(y_{0}-s\right)$ of the amount $y_{0}-s$ resulted by the transfer of $s$ in the second period;

(b) the probabilistic expected value $M(v((1+r) s+\tilde{y}))$, resulted in the second period from the uncertain income $\tilde{y}$ and from the amount $(1+r) s$.

The transformation of the above model in a possibilistic one is done by:

- the uncertain income from period 1 will no longer be a random variable $\tilde{y}$, but a fuzzy number $A$;

- to define the total utility of the model it is necessary to have a concept of "possibilistic expected utility".

Using this thesis, we will define an optimal saving model in the context offered by the expected utility operators.

We fix a weighting function $f:[0,1] \rightarrow \mathbf{R}$ and an expected utility operator $T$. The possibilistic optimal saving model associated with $T$ has the following initial data:

- $\quad$ Two utility functions $u$ and $v$, verifying the conditions from the probabilistic model;

- a sure income in period 0 and an uncertain income in period 1 , described by a fuzzy number $A$ whose level sets are $[A]^{\gamma}=\left[a_{1}(\gamma), a_{2}(\gamma)\right], \gamma \in[0,1]$.

- $\quad s$ is the level of saving for period 0 ;

- $\quad r$ is the interest rate for saving.

The uncertain income from period 1 will be interpreted as a labor income risk (analogous to [6]). The total utility function of the model will be:

$$
V_{T}(s)=u\left(y_{0}-s\right)+T(A, v((1+r) s+y))
$$

One notices the way the definition of $V_{T}(s)$ is inspired from (6): Instead of $M(v((1+r) s+\tilde{y}))$ appears the generalized possibilistic expected utility $T(A, v((1+r) s+y))$.

In case of the model described above, the consumer will choose the level of $s$ resulted from solving the following optimization problem:

$$
\max _{s} V_{T}(s)
$$

To study the existence and the properties of the solutions of (8), we will assume that $T$ is a $D$-operator. 
Proposition 4. If $T$ is a D-operator then

(i) $V_{T}^{\prime}(s)=-u^{\prime}\left(y_{0}-s\right)+(1+r) T\left(A, v^{\prime}((1+r) s+y)\right)$

(ii) $V_{T}^{\prime \prime}(s)=u^{\prime \prime}\left(y_{0}-s\right)+(1+r)^{2} T\left(A, v^{\prime \prime}((1+r) s+y)\right)$

(iii) $V_{T}$ is a concave function.

\section{Proof.}

(i) Taking into account the axiom $\left(D_{2}\right)$ from Definition 2 and condition (c) of Definition 1 the following equalities are true:

$$
\begin{aligned}
V_{T}^{\prime}(s) & =-u^{\prime}\left(y_{0}-s\right)+\frac{d}{d s} T(A, v((1+r) s+y)) \\
& =-u^{\prime}\left(y_{0}-s\right)+T\left(A, \frac{d}{d s} v((1+r) s+y)\right) \\
& =-u^{\prime}\left(y_{0}-s\right)+T\left(A,(1+r) v^{\prime}((1+r) s+y)\right) \\
& =-u^{\prime}\left(y_{0}-s\right)+(1+r) T\left(A, v^{\prime}((1+r) s+y)\right)
\end{aligned}
$$

(ii) Analogous with the proof of (i).

(iii) We recall the hypotheses: $u^{\prime \prime}<0, v^{\prime \prime}<0$. Then, by condition (d) of Definition 1 we have $T\left(A, v^{\prime \prime}((1+r) s+y)\right) \leq 0$, thus $V_{T}^{\prime \prime}(s) \leq 0$.

We notice that in the proof of the previous proposition it is the first time when we use the hypothesis that $T$ is a $D$-operator.

By the previous proposition, we can consider the solution $s_{T}^{*}$ of the optimization problem (8). Taking into account Proposition 4 (i), the first-order condition associated with the optimization problem (8) will be written as:

$$
u^{\prime}\left(y_{0}-s\right)=(1+r) T\left(A, v^{\prime}((1+r) s+y)\right)
$$

We will assume now that the supplementary condition is fulfilled: $v$ is of class $C^{3}$. Applying Proposition 1 we will have the approximation:

$$
T\left(A, v^{\prime}((1+r) s+y)\right) \approx v^{\prime}\left(E_{f}((1+r) s+A)\right)+\frac{1}{2} v^{\prime \prime \prime}\left((1+r) s+E_{f}(A)\right) \operatorname{Var}_{T}(A)
$$

Then the first-order condition (9) will be written as:

$$
\left.u^{\prime}\left(y_{0}-s\right) \approx(1+r)\left[v^{\prime}\left((1+r) s+E_{f}(A)\right)\right)+\frac{1}{2} v^{\prime \prime \prime}\left((1+r) s+E_{f}(A)\right) \operatorname{Var}_{T}(A)\right]
$$

The solution $s_{T}^{*}=s_{T}^{*}(A)$ of the optimization problem (8) will be called the " $T$-optimal saving", and the above model will be called the " $T$-model of saving".

\section{The Approximate Computation of the T-Optimal Saving}

In this section we will prove a formula for the approximate computation of the optimal saving level defined for the saving model (8).

The $T$-optimal saving $s_{T}^{*}$ verifies the first-order condition (9):

$$
u^{\prime}\left(y_{0}-s_{T}^{*}\right)=(1+r) T\left(A, v^{\prime}\left((1+r) s_{T}^{*}+y\right)\right)
$$

Since to determine the exact solution $s_{T}^{*}$ of the Equation (6) is difficult, we will look for approximation formulas.

We will assume that $u$ is of class $C^{2}$ and $v$ is of class $C^{4}$. Then, by the first-order condition (10), the following relation is true:

$$
u^{\prime}\left(y_{0}-s_{T}^{*}\right) \approx(1+r)\left[v^{\prime}\left((1+r) s_{T}^{*}+E_{f}(A)\right)+\frac{1}{2} v^{\prime \prime \prime}\left((1+r) s_{T}^{*}+E_{f}(A) \operatorname{Var}_{T}(A)\right)\right]
$$


Proposition 5. An approximate value of $s_{T}^{*}$ is given by the following formula:

$$
s_{T}^{*} \approx \frac{u^{\prime}\left(y_{0}\right)-(1+r)\left[v^{\prime}\left(E_{f}(A)\right)+\frac{1}{2} v^{\prime \prime \prime}\left(E_{f}(A)\right) \operatorname{Var}_{T}(A)\right]}{u^{\prime \prime}\left(y_{0}\right)+(1+r)^{2}\left[v^{\prime \prime}\left(E_{f}(A)\right)+\frac{1}{2} v^{i v}\left(E_{f}(A)\right) \operatorname{Var}_{T}(A)\right]}
$$

Proof. With the notation $a=E_{f}(A)$ and the following Taylor series approximations:

$$
\begin{aligned}
u^{\prime}\left(y_{0}-s_{T}^{*}\right) & \approx u^{\prime}\left(y_{0}\right)-s_{T}^{*} u^{\prime \prime}\left(y_{0}\right) \\
v^{\prime}\left((1+r) s_{T}^{*}+a\right) & \approx v^{\prime}(a)+(1+r) s_{T}^{*} v^{\prime \prime}(a) \\
v^{\prime \prime \prime}\left((1+r) s_{T}^{*}+a\right) & \approx v^{\prime \prime \prime}(a)+(1+r) s_{T}^{*} v^{i v}(a)
\end{aligned}
$$

therefore replacing them in (12), the following relation follows:

$$
u^{\prime}\left(y_{0}\right)-s_{T}^{*} u^{\prime \prime}\left(y_{0}\right) \approx(1+r)\left[v^{\prime}(a)+(1+r) s_{T}^{*} v^{\prime \prime}(a)+\frac{1}{2}\left(v^{\prime \prime \prime}(a)+(1+r) s_{T}^{*} v^{i v}(a)\right) \operatorname{Var}_{T}(A)\right]
$$

which can be written as:

$$
\begin{aligned}
& u^{\prime}\left(y_{0}\right)-s_{T}^{*} u^{\prime \prime}\left(y_{0}\right) \approx(1+r)\left[v^{\prime}(a)+\frac{1}{2} v^{\prime \prime \prime}(a) \operatorname{Var}_{T}(A)\right]+ \\
& +(1+r)^{2} s_{T}^{*}\left[v^{\prime \prime}(a)+\frac{1}{2} v^{i v}(a) \operatorname{Var}_{T}(A)\right]
\end{aligned}
$$

Computing $s_{T}^{*}$ from the previous relation we will obtain (13).

Remark 1. Applying Proposition 1 the following approximations follow:

$$
\begin{aligned}
& T\left(A, v^{\prime}\right) \approx v^{\prime}(a)+\frac{1}{2} v^{\prime \prime \prime}(a) \operatorname{Var}_{T}(A) \\
& T\left(A, v^{\prime \prime}\right) \approx v^{\prime \prime}(a)+\frac{1}{2} v^{i v}(a) \operatorname{Var}_{T}(A)
\end{aligned}
$$

Then the approximation Formula (13) can be written in a more concentrated form:

$$
s_{T}^{*} \approx \frac{u^{\prime}\left(y_{0}\right)-(1+r) T\left(A, v^{\prime}\right)}{u^{\prime \prime}\left(y_{0}\right)+(1+r)^{2} T\left(A, v^{\prime \prime}\right)}
$$

By means of the approximation Formulas (11) or (14), the consumer will establish the optimal level of the amount $s$ transferred from period 0 in period 1 to minimize the effect of possibilistic risk.

Let $S, T$ be two operators. We define $S \leq T$ if $S(A, g) \leq T(A, g)$ for any fuzzy number $A$ and for any continuous function $g: \mathbf{R} \rightarrow \mathbf{R}$. One easily checks that $\leq$ is a partial order on the set of expected utility operators. We consider the $S$-model of saving and the $T$-model of saving (both defined with the data from Section 4) and the optimal saving levels $\alpha_{S}^{*}, \alpha_{T}^{*}$ corrsponding to the two models.

Proposition 6. Let $S, T$ be two D-operators. If $S \leq T$ then $\alpha_{S}^{*} \geq \alpha_{T}^{*}$.

Proof. We recall the hypotheses from the construction of the saving models: $u^{\prime}<0, v^{\prime}<0, u^{\prime \prime}>0$, $v^{\prime \prime}>0$. Then, by the hypothesis $S \leq T$ and by applying axiom (d) from Definition 1 the following results will follow:

$$
S\left(A, v^{\prime}\right) \leq T\left(A, v^{\prime}\right) \leq 0 ; T\left(A, v^{\prime \prime}\right) \operatorname{lgeqS}\left(A, v^{\prime \prime}\right) \geq 0
$$

From the first inequality one obtains $-(1+r) S\left(A, v^{\prime}\right) \geq-(1+r) T\left(A, v^{\prime}\right)$, thus

$$
u^{\prime}\left(y_{0}\right)-(1+r) S\left(A, v^{\prime}\right) \geq u^{\prime}\left(y_{0}\right)-(1+r) T\left(A, v^{\prime}\right)
$$

Analogously, from $T\left(A, v^{\prime \prime}\right) \geq S\left(A, v^{\prime \prime}\right) \geq 0$ and from $u^{\prime \prime}\left(y_{0}\right)>0$ the following inequality follows: 


$$
\frac{1}{u^{\prime \prime}\left(y_{0}\right)+(1+r)^{2} S\left(A, v^{\prime \prime}\right)} \geq \frac{1}{u^{\prime \prime}\left(y_{0}\right)+(1+r)^{2} T\left(A, v^{\prime \prime}\right)}>0
$$

Multiplying inequalities (15) and (16) it follows that

$$
\frac{u^{\prime}\left(y_{0}\right)-(1+r) S\left(A, v^{\prime}\right)}{u^{\prime \prime}\left(y_{0}\right)+(1+r)^{2} S\left(a, v^{\prime \prime}\right)} \geq \frac{u^{\prime}\left(y_{0}\right)-(1+r) T\left(A, v^{\prime}\right)}{u^{\prime \prime}\left(y_{0}\right)+(1+r)^{2} T\left(A, v^{\prime \prime}\right)}
$$

Taking into account the approximation Formula (14) applied to the $D$-operators $S$ and $T$, the inequality (17) becomes $\alpha_{S}^{*} \geq \alpha_{T}^{*}$.

\section{The T-Precautionary Saving}

In the model of $T$-optimal saving from Section 4 risk appears in the second period as the fuzzy number $A$. In this section we will associate with the risk model (8) a model in which risk no longer appears.

We will modify the model of T-optimal saving in the following way: In period 1, the income will be the possibilistic expected value $E_{f}(A)$. The other parameters of the first model will be kept for the second one. Then the total utility function of the second model will be:

$$
W(s)=u\left(y_{0}-s\right)+v\left((1+r) s+E_{f}(A)\right)
$$

and the optimization problem will be:

$$
\max _{s} W(s)
$$

We recall that the utility functions $u, v$ are of class $C^{2}$ and $u^{\prime}>0, v^{\prime}>0, u^{\prime \prime}<0, v^{\prime \prime}<0$. One notices that

$$
\begin{aligned}
& W^{\prime}(s)=-u^{\prime}\left(y_{0}-s\right)+(1+r) v^{\prime}\left((1+r) s+E_{f}(A)\right) \\
& W^{\prime \prime}(s)=u^{\prime \prime}\left(y_{0}-s\right)+(1+r)^{2} v^{\prime \prime}\left((1+r) s+E_{f}(A)\right)
\end{aligned}
$$

from where it follows that $W^{\prime \prime}(s) \leq 0$, thus the total utility function $W(s)$ is concave. Accordingly we can consider the solution $s^{*}=s^{*}(A)$ of the optimization problem (19). By (20), the first-order condition $W^{\prime}\left(s^{*}\right)=0$ will be written as:

$$
u^{\prime}\left(y_{0}-s^{*}\right)=(1+r) v^{\prime}\left((1+r) s^{*}+E_{f}(A)\right)
$$

Up to now we deal with two models of optimal saving: The possibilistic model (8), in which the risk is a fuzzy number $A$ and the deterministic model (22), in which the risk $A$ has been replaced by the posibilistic expected value $E_{f}(A)$.

Proposition 7. An approximate value of the optimal saving s* corresponding to the uncertain model (22) is given by:

$$
s^{*} \approx \frac{u^{\prime}\left(y_{0}\right)-(1+r) v^{\prime}(a)}{u^{\prime \prime}\left(y_{0}\right)+(1+r)^{2} v^{\prime \prime}(a)}
$$

where $a=E_{f}(A)$.

Proof. The approximation Formula (23) is obtained using the Taylor approximation and the same steps from the proof of Proposition 5.

The study of the optimal saving models (8) and (22) will be done in parallel. For example, one poses the question of comparing the optimal saving levels $s_{T}^{*}$ and $s^{*}$ : On what conditions the substitution of the certain value $E_{f}(A)$ with a risk $A$ leads to an increase or a decrease in optimal saving. 
The difference $s_{T}^{*}-s^{*}$ will be called "T-precautionary saving". This indicator measures the variation of optimal saving when we pass from the model without risk (19) to the model with risk (8). In other words, the $T$-precautionary saving $s_{T}^{*}-s^{*}$ shows to which extent the optimal saving level increases or decreases in the presence of possibilistic risk $A$.

Proposition 8. Assume that $v$ is of class $C^{3}$. If $v^{\prime \prime \prime} \geq 0$ then for any fuzzy number $A$, we have $s_{T}^{*}(A)-$ $s^{*}(A) \geq 0$.

Proof. By Formulas (11) and (20), the following equalities hold

$$
\begin{aligned}
W^{\prime}\left(s_{T}^{*}\right) & =-u^{\prime}\left(y_{0}-s_{T}^{*}\right)+(1+r) v^{\prime}\left((1+r) s_{T}^{*}+E_{f}(A)\right) \\
& -(1+r) T\left(A, v^{\prime}\left((1+r) s_{T}^{*}+y\right)\right)+(1+r) v^{\prime}\left((1+r) s_{T}^{*}+E_{f}(A)\right) \\
& =(1+r)\left[v^{\prime}\left((1+r) s_{T}^{*}+E_{f}(A)\right)-T\left(A, v^{\prime}\left((1+r) s_{T}^{*}+y\right)\right)\right.
\end{aligned}
$$

Taking into account that $E_{f}\left((1+r) s_{T}^{*}+A\right)=(1+r) s_{T}^{*}+E_{f}(A)$ it follows:

$$
W^{\prime}\left(s_{T}^{*}\right)=(1+r)\left[v^{\prime}\left(E_{f}\left((1+r) s_{T}^{*}+A\right)\right)-T\left(A, v^{\prime}\left((1+r) s_{T}^{*}+y\right)\right)\right]
$$

Since $W^{\prime}$ is a strictly decreasing function, the following equivalences are true:

$$
\begin{aligned}
s_{T}^{*} \geq s^{*} & \text { iff } W^{\prime}\left(s_{T}^{*}\right) \leq W^{\prime}\left(s^{*}\right)=0 \\
& \text { iff }(1+r)\left[v^{\prime}\left(E_{f}\left((1+r) s_{T}^{*}+A\right)\right)-T\left(A, v^{\prime}\left((1+r) s_{T}^{*}+y\right)\right)\right] \leq 0 \\
& \text { iff } v^{\prime}\left(E_{f}\left((1+r) s_{T}^{*}+A\right)\right) \leq T\left(A, v^{\prime}\left((1+r) s_{T}^{*}+y\right)\right) .
\end{aligned}
$$

By hypothesis $v^{\prime \prime \prime} \geq 0$, thus $v^{\prime}$ is a convex function. Applying Proposition 3 it follows the inequality $v^{\prime}\left(E_{f}\left((1+r) s_{T}^{*}+A\right)\right) \leq T\left(A, v^{\prime}\left((1+r) s_{T}^{*}+y\right)\right.$. Then, by the equivalences above, one tobtains $s_{T}^{*}-s^{*} \geq 0$.

Remark 2. The inequality $s_{T}^{*}(A) \geq s^{*}(A)$ asserts that in the presence of a possibilistic risk $A$, the optimal saving level increases. In other words, condition $s_{T}^{*}(A) \geq s^{*}(A)$ reflects an attitude of prudence of the consumer in front of a risk $A$ : The existence of risk makes the consumer raise the optimal saving level from $s^{*}(A)$ to $s_{T}^{*}(A)$.

The discussion from Remark 2 suggests the following notion of "possibilistic prudence" associated with an expected utility operator $T$.

Definition 3. Let $T$ be an expected utility operator. Then the consumer $(u, v)$ is T-prudent if for any fuzzy number A representing risk, the following inequality holds: $s_{T}^{*}(A) \geq s^{*}(A)$.

By Proposition 8 , the inequality $v^{\prime \prime \prime} \geq 0$ is a sufficient condition for the consumer's $T$-prudence. Naturally one poses the question whether $v^{\prime \prime \prime} \geq 0$ is also a necessary condition for T-prudence. The problem is important since $v^{\prime \prime \prime} \geq 0$ is a necessary and sufficient condition for prudence defined in a probabilistic sense (see Kimball [23] and [5]).

For the $D$-operator $T_{1}$ defined in Example 1, the inequality $v^{\prime \prime \prime} \geq 0$ is a necessary and sufficient condition of $T_{1}$-prudence (by [20], Theorem 1).

The characterization of $T$-prudence by the property $v^{\prime \prime \prime}>0$ remains an open problem in case of an arbitrary $D$-operator $T$.

\section{Particular Cases and Examples}

In this section we will study the $T$-optimal saving for some particular $D$-operators, making the following assumptions on the weighting function $f$ and the fuzzy number $A$ :

- $f(t)=2 t$, for any $t \in[0,1]$;

- $A$ is the triangular fuzzy number $(b, \alpha, \beta)$ : 


$$
A(t)= \begin{cases}1-\frac{b-t}{\alpha} & b-\alpha \leq t \leq b \\ 1-\frac{t-b}{\beta} & b \leq t \leq b+\beta \\ 0 & \text { otherwise }\end{cases}
$$

As to the $D$-operator $T$, we will consider the following particular cases:

(a) $T$ is the $D$-operator $T_{1}$. By [12], Examples 3.3.10 and 3.4.10 we have the following expressions for the possibilistic expected values $E_{f}(A)$ and for the $T_{1}$-variance $\operatorname{Var}_{T_{1}}(A)$.

$$
\begin{gathered}
a=E_{f}(A)=b+\frac{\beta-\alpha}{6} \\
\operatorname{Var}_{T_{1}}(A)=\frac{\alpha^{2}+\beta^{2}+\alpha \beta}{18}
\end{gathered}
$$

Replacing $\operatorname{Var}_{T_{1}}(A)$ in (13), The $T_{1}$-optimal saving level becomes:

$$
s_{T 1}^{*} \approx \frac{u^{\prime}\left(y_{0}\right)-(1+r)\left[v^{\prime}(a)+\frac{1}{2} v^{\prime \prime \prime}(a) \frac{\alpha^{2}+\beta^{2}+\alpha \beta}{18}\right]}{u^{\prime \prime}\left(y_{0}\right)+(1+r)^{2}\left[v^{\prime \prime}(a)+\frac{1}{2} v^{i v}(a) \frac{\alpha^{2}+\beta^{2}+\alpha \beta}{18}\right]}
$$

(b) $T$ is the D-operator $T_{2}$. By [12], Example 3.4.10 we have

$$
\operatorname{Var}_{T_{2}}(A)=\frac{\alpha^{2}+\beta^{2}}{36}
$$

Replacing $\operatorname{Var}_{T_{2}}(A)$ in (13), the $T_{2}$-optimal saving level becomes:

$$
s_{T 2}^{*} \approx \frac{u^{\prime}\left(y_{0}\right)-(1+r)\left[v^{\prime}(a)+\frac{1}{2} v^{\prime \prime \prime}(a) \frac{\alpha^{2}+\beta^{2}}{36}\right]}{u^{\prime \prime}\left(y_{0}\right)+(1+r)^{2}\left[v^{\prime \prime}(a)+\frac{1}{2} v^{i v}(a) \frac{\alpha^{2}+\beta^{2}}{36}\right]}
$$

(c) According to [12], Proposition 5.1.5, one can consider the $D$-operator $U=\frac{1}{2} T_{1}+\frac{1}{2} T_{2}$. Applying Proposition 5.1.6. from [12] and Formulas (25) and (27) it follows:

$$
\operatorname{Var}_{U}(A)=\frac{1}{2} \operatorname{Var}_{T_{1}}(A)+\frac{1}{2} \operatorname{Var}_{T_{2}}(A)=\frac{3 \alpha^{2}+3 \beta^{2}+2 \alpha \beta}{72}
$$

The optimal $U$-optimal saving level becomes:

$$
s_{U}^{*} \approx \frac{u^{\prime}\left(y_{0}\right)-(1+r)\left[v^{\prime}(a)+\frac{1}{2} v^{\prime \prime \prime}(a) \frac{3 \alpha^{2}+3 \beta^{2}+2 \alpha \beta}{72}\right]}{u^{\prime \prime}\left(y_{0}\right)+(1+r)^{2}\left[v^{\prime \prime}(a)+\frac{1}{2} v^{i v}(a) \frac{3 \alpha^{2}+3 \beta^{2}+2 \alpha \beta}{72}\right]}
$$

If $A$ is a symmetric triangular fuzzy number $(b, \alpha)$, then, setting $\beta=\alpha$ in (26), (28) and (29) we find the following forms of the three $T$-optimal saving levels:

$$
\begin{aligned}
& s_{T 1}^{*} \approx \frac{u^{\prime}\left(y_{0}\right)-(1+r)\left[v^{\prime}(a)+v^{\prime \prime \prime}(a) \frac{\alpha^{2}}{12}\right]}{u^{\prime \prime}\left(y_{0}\right)+(1+r)^{2}\left[v^{\prime \prime}(a)+\frac{1}{2} v^{i v}(a) \frac{\alpha^{2}}{12}\right]} ; \\
& s_{T 2}^{*} \approx \frac{u^{\prime}\left(y_{0}\right)-(1+r)\left[v^{\prime}(a)+v^{\prime \prime \prime}(b) \frac{\alpha^{2}}{36}\right]}{u^{\prime \prime}\left(y_{0}\right)+(1+r)^{2}\left[v^{\prime \prime}(a)+\frac{1}{2} v^{i v}(a) \frac{\alpha^{2}}{36}\right]} ; \\
& s_{U}^{*} \approx \frac{u^{\prime}\left(y_{0}\right)-(1+r)\left[v^{\prime}(a)+\frac{\alpha^{2}}{18}\right]}{u^{\prime \prime}\left(y_{0}\right)+(1+r)^{2}\left[v^{\prime \prime}(a)+\frac{1}{2} v^{i v}(a) \frac{\alpha^{2}}{18}\right]}
\end{aligned}
$$

Example 3. Assume that $A$ is the triangular fuzzy number $A=(b, \alpha, \beta)$ and the utility functions $u, v$ are CRRA-type: 


$$
u(w)=v(w)= \begin{cases}\frac{w^{1-\gamma}}{1-\gamma} & \gamma>1 \\ \ln (w) & \gamma=1\end{cases}
$$

Then we have the cases:

- $\quad$ for $\gamma>1$ :

$u^{\prime}(w)=\frac{w^{1-\gamma}}{1-\gamma}, u^{\prime}(w)=w^{-\gamma}, u^{\prime \prime}(w)=(-\gamma) w^{-\gamma-1}, u^{\prime \prime \prime}(w)=\gamma(\gamma+1) w^{-\gamma-2}, u^{i v}=-\gamma(\gamma+$ 1) $(\gamma+2) w^{-\gamma-3}$.

The optimal saving levels become:

$$
\begin{aligned}
& s_{T 1}^{*} \approx \frac{y_{0}^{-\gamma}-(1+r)\left[a^{-\gamma}+\frac{1}{2} \gamma(\gamma+1) a^{\left.-\gamma-2 \frac{\alpha^{2}+\beta^{2}+\alpha \beta}{18}\right]}\right.}{(-\gamma) y_{0}^{-\gamma-1}+(1+r)^{2}\left[(-\gamma) a^{-\gamma-1}-\frac{1}{2} \gamma(\gamma+1)(\gamma+2) a^{-\gamma-3} \frac{\alpha^{2}+\beta^{2}+\alpha \beta}{18}\right]} ; \\
& s_{T 2}^{*} \approx \frac{y_{0}^{-\gamma}-(1+r)\left[a^{-\gamma}+\frac{1}{2} \gamma(\gamma+1) a^{\left.-\gamma-2 \frac{\alpha^{2}+\beta^{2}}{36}\right]}\right.}{(-\gamma) y_{0}^{-\gamma-1}+(1+r)^{2}\left[(-\gamma) a^{-\gamma-1}-\frac{1}{2} \gamma(\gamma+1)(\gamma+2) a^{-\gamma-3} \frac{\alpha^{2}+\beta^{2}}{36}\right]} \\
& s_{U}^{*} \approx \frac{y_{0}^{-\gamma}-(1+r)\left[a^{-\gamma}+\frac{1}{2} \gamma(\gamma+1) a^{-\gamma-2} \frac{3 \alpha^{2}+3 \beta^{2}+2 \alpha \beta}{72}\right]}{(-\gamma) y_{0}^{-\gamma-1}+(1+r)^{2}\left[(-\gamma) a^{-\gamma-1}-\frac{1}{2} \gamma(\gamma+1)(\gamma+2) a^{-\gamma-3} \frac{3 \alpha^{2}+3 \beta^{2}+2 \alpha \beta}{72}\right]}
\end{aligned}
$$

- $\quad$ for $\gamma=1$ :

$u^{\prime}(w)=\frac{1}{w}, u^{\prime \prime}(w)=-\frac{1}{w^{2}}, u^{\prime \prime \prime}(w)=\frac{2}{w^{3}}, u^{i v}(w)=\frac{-3}{w^{4}}$.

The optimal saving levels become:

$$
\begin{gathered}
s_{T 1}^{*} \approx \frac{y_{0}^{-1}-(1+r)\left[a^{-1}+a^{-3} \frac{\alpha^{2}+\beta^{2}+\alpha \beta}{18}\right]}{y_{0}^{-2}+(1+r)^{2}\left[a^{-2}-3 a^{-4} \frac{\alpha^{2}+\beta^{2}+\alpha \beta}{18}\right]} ; \\
s_{T 2}^{*} \approx \frac{y_{0}^{-1}-(1+r)\left[a^{-1}+a^{-3} \frac{\alpha^{2}+\beta^{2}}{36}\right]}{y_{0}^{-2}+(1+r)^{2}\left[a^{-2}-3 a^{-4} \frac{\alpha^{2}+\beta^{2}}{36}\right]} \\
s_{U}^{*} \approx \frac{y_{0}^{-1}-(1+r)\left[a^{-1}+a^{-3} \frac{3 \alpha^{2}+3 \beta^{2}+2 \alpha \beta}{72}\right]}{y_{0}^{-2}+(1+r)^{2}\left[a^{-2}-3 a^{-4} \frac{3 \alpha^{2}+3 \beta^{2}+2 \alpha \beta}{72}\right]} .
\end{gathered}
$$

\section{Concluding Remarks}

In this paper a possibilistic saving model is atudied, in which the labor-income risk is modeled by a fuzzy number.

The paper contains the following contributions:

- the construction of a saving model in the possibilistic framework offered by the expected utility operators;

- $\quad$ the use of $D$-operators (a class of expected utility operators) in the analysis of the solution of the optimization problem associated with the model (existence, uniqueness and its approximate calculation);

- $\quad$ getting some approximation formula of the optimal saving models in a few important cases.

We indicate now a few directions of future research deriving from the paper:

(a) To determine a class of expected utility operators allowing to establish some necessary and sufficient conditions of extra saving (in the framework of associated saving models); 
(b) the use of expected utility operators to study saving models with other types of risk: Wealth risk, asset risk and capital risk (by [24,25]);

(c) the study of optimal saving with several risk parameters: Risk may be represented by a possibilistic vector (the components are fuzzy numbers) or by a mixed vector (some components are fuzzy numbers, others are random variables) (by [12], Chapters 6 and 7);

(d) to apply the results from the paper for possibilistic saving models built from a dataset, using the sample percentile method of Vercher et al. [26].

Author Contributions: Conceptualization, I. G. ; methodology, J.K.; validation, I.G., J.K.; formal analysis, I.G.; investigation, I.G.; writing-original draft preparation, I.G.; writing-review and editing, I.G.; visualization, J.K.; supervision, I.G.. All authors have read and agreed to the published version of the manuscript.

Funding: This research received no external funding.

Acknowledgments: The authors would like to thank the two reviewers for their remarks and comments which led to an improved version of the paper.

Conflicts of Interest: The authors declare no conflict of interest.

\section{References}

1. Leland, H. Saving and uncertainty: The precautionary demand for saving. Q. J. Econ. 1968, 82, 465-473. [CrossRef]

2. Sandmo, A. The effect of uncertainty on saving decisions. Rev. Econ. Stud., 1970, 37, 353-360. [CrossRef]

3. Drèze, J.; Modigliani, F. Consumption decision under uncertainty. J. Econ. Theory 1972, 5, 308-355.

4. Rothschild, M.; Stiglitz, E. Increasing risk II: Economic Consequences. J. Econ. Theory 1971, 3, 66-84. [CrossRef]

5. Kimball, M.S. Precautionary Motives for Holding Assets, New Palgrave Dictionary of Money and Finance; MacMillan Press: London, UK; Stockton Publishers: New York, NY, USA, 1992; Volume 3, pp. 158-161.

6. Eekhoudt, L.; Schlesinger, H. Changes in risk and the demand for saving. J. Monet. Econ. 2008, 55, 1329-1336. [CrossRef]

7. Li, J. Precautionary saving in the presence of labor income and interest rate risks. J. Econ. 1968, 82, 251-266. [CrossRef]

8. Baiardi, D.; Magnani, M.; Menegatti, M. Precautionary saving under many risks. J. Econ. 2014, 113, $211-228$. [CrossRef]

9. Eeckhoudt, L.; Gollier, C.; Schlesinger, H. Economic and Financial Decision under Risk; Princeton University Press: Princeton, NJ, USA, 2005.

10. Baiardi, D.; Magnani, M.; Menegatti, M. The theory of precautionary saving: An overview of recent developments. Rev. Econ. Househ. 2019. [CrossRef]

11. Zadeh, L.A. Fuzzy sets as a basis for a theory of possibility. Fuzzy Sets Syst. 1978, 1, 3-28. [CrossRef]

12. Georgescu, I. Possibility Theory and the Risk; Springer: Berlin, Germany, 2012.

13. Carlsson, C.; Fullér, R. Possibility for Decision; Springer: Berlin, Germany, 2011.

14. Dubois, D.; Prade, H. Possibility Theory; Plenum Press: New York, NY, USA, 1988.

15. Carlsson, C.; Fullér, R. On possibilistic mean value and variance of fuzzy numbers. Fuzzy Sets Syst. 2001, 123, 315-326. [CrossRef]

16. Majlender, P. A Normative Approach to Possibility Theory and Decision Support. Ph.D. Thesis, Turku Centre for Computer Science, Turku, Finland, 2004.

17. Mezei, J. A Quantitative View on Fuzzy Numbers. Ph.D. Thesis, Turku Centre for Computer Science, Turku, Finland, 2011.

18. Collan, M.; Fedrizzi, M.; Luukka, P. Possibilistic risk aversion in group decisions: Theory with application in the insurance of giga-investments valued through the fuzzy pay-off method. Soft Comput. 2017, 24, 4375-4386. [CrossRef]

19. Georgescu, I.; Kinnunen, J. Precautionary saving with possibilistic background risk. In Proceedings of the CINTI 2015, Budapest, Hungary, 19-21 November 2015; pp. 165-169. 
20. Lucia-Casademunt, A.M.; Georgescu, I. Connecting possibilistic prudence and optimal saving. Int. J. Interact. Multimed. Artif. Intell. 2013, 2, 38-45.

21. Georgescu, I. Expected utility operators and possibilistic risk aversion. Soft Comput. 2012, 16, 1671-1680. [CrossRef]

22. Georgescu, I.; Fono, L.A. A portfolio choice problem in the framework of expected utility operators. Mathematics 2019, 7, 669. [CrossRef]

23. Kimball, M.S. Precautionary saving in the small and in the large. Econometrica 1990, 58, 53-73. [CrossRef]

24. Gunning, J.W. Risk and saving: A taxonomy. Econ. Lett. 2010, 107, 39-41. [CrossRef]

25. Vergara, M. Precautionary saving: A taxonomy of prudence. Econ. Lett. 2007, 15, 18-20. [CrossRef]

26. Vercher, E.; Bermudez, J.D.; Segura, J.V. Fuzzy Portfolio Optimization under Downside Risk Measures. Fuzzy Sets Syst. 2007, 158, 769-782. [CrossRef]

(C) 2020 by the authors. Licensee MDPI, Basel, Switzerland. This article is an open access article distributed under the terms and conditions of the Creative Commons Attribution (CC BY) license (http:/ / creativecommons.org/licenses/by/4.0/). 